\title{
Genetic determinants of Pseudomonas aeruginosa biofilm establishment
}

\section{Correspondence \\ Susanne Häussler susanne.haeussler@ helmholtz-hzi.de}

Received 3 August 2009

Revised 7 October 2009

Accepted 19 October 2009

\author{
Mathias Müsken, ${ }^{1}$ Stefano Di Fiore, ${ }^{2,3}$ Andreas Dötsch, ${ }^{1}$ Rainer Fischer ${ }^{3}$ \\ and Susanne Häussler ${ }^{1,4}$
}

${ }^{1}$ Chronic Pseudomonas Infection Research Group, Helmholtz Center for Infection Research, Inhoffenstrasse 7, D-38124 Braunschweig, Germany

${ }^{2}$ Institute for Molecular Biotechnology, RWTH Aachen University, Worringerweg 1, D-52704 Aachen, Germany

${ }^{3}$ Fraunhofer Institute for Molecular Biology and Applied Ecology, Forckenbeck Str. 6, D-52704 Aachen, Germany

${ }^{4}$ Twincore, Center for Experimental and Clinical Infection Research, a joint venture of the Helmholtz Center for Infection Research and the Medical School Hannover, Feodor Lynen Strasse 7, D-30265 Hannover, Germany

The establishment of bacterial biofilms on surfaces is a complex process that requires various factors for each consecutive developmental step. Here we report the screening of the comprehensive Harvard Pseudomonas aeruginosa PA14 mutant library for mutants exhibiting an altered biofilm phenotype. We analysed the capability of all mutants to form biofilms at the bottom of a 96-well plate by the use of an automated confocal laser-scanning microscope and found 394 and 285 genetic determinants of reduced and enhanced biofilm production, respectively. Overall, $67 \%$ of the identified mutants were affected within genes encoding hypothetical proteins, indicating that novel developmental pathways are likely to be dissected in the future. Nevertheless, a common theme that emerged from the analysis of the genes with a predicted function is that the establishment of a biofilm requires regulatory components that are involved in survival under microaerophilic growth conditions, arginine metabolism, alkyl-quinolone signalling, $\mathrm{pH}$ homeostasis and the DNA repair system.

\section{INTRODUCTION}

In most environments bacteria live primarily in sessile communities, referred to as biofilms (Costerton et al., 1995). Biofilms form on a number of surfaces, including tissues of the human host, where they represent an important determinant of chronic infections (Gilbert et al., 2002). Biofilm infections increasingly pose a challenge to modern medicine, because they are rarely eradicated due to the inherent resistance of biofilms to current antimicrobial therapies (Gilbert \& McBain, 2001).

The opportunistic pathogens Staphylococcus epidermidis and Staphylococcus aureus, as well as Pseudomonas aeruginosa, rank among the clinically most significant organisms that form biofilms, and have become the model organisms for studying Gram-positive and Gram-negative

Abbreviations: ADI pathway, arginine deaminase pathway; CF, cystic fibrosis; NA, numerical aperture; PI, propidium iodide.

Two supplementary tables, showing PA14 mutants exhibiting reduced biofilm formation and PA14 mutants exhibiting enhanced biofilm formation as compared with the wild-type, are available with the online version of this paper biofilms, respectively. These pathogens cause severe nosocomial infections in the immunocompromised host, and $P$. aeruginosa is the most dominant fate-determining pathogen in cystic fibrosis (CF) (Tümmler et al., 1997) as well as a serious problem in individuals with burns. Despite the high incidence of chronic persistent biofilm infections and their significant morbidity, an understanding of the molecular mechanisms underlying the establishment of a structured lifestyle in bacterial communities remains limited.

Biofilms are formed from individual planktonic cells in a complex and presumably highly regulated developmental process, which has been observed microscopically in a number of bacteria, and which involves a coordinated sequence of events that include primary surface attachment, microcolony formation, maturation, expansion, and finally dissemination. The use of simple high-throughput screening assays for biofilm development has greatly facilitated the analysis of the genetic elements involved in this process. For instance, by growing P. aeruginosa PA14 transposon mutants under static conditions in the wells of microtitre plates and screening for surface attachment deficient (sad) mutants, O'Toole and Kolter showed that 
flagella are required for biofilm formation, and type IV pili for biofilm and microcolony formation (O'Toole \& Kolter, 1998a).

Since then, many additional factors have been reported to influence biofilm formation in $P$. aeruginosa at different developmental stages. Biofilm formation has been shown to be largely dependent on the culture conditions, which can influence the requirement for a particular factor. For example, the requirement for flagella-driven motility for initial bacterial adherence depends on the carbon source (Klausen et al., 2003), iron limitation necessitates the presence of the siderophore pyoverdine to form biofilms (Patriquin et al., 2008), and the presence of the glycoprotein mucin supports the establishment of structured $P$. aeruginosa communities (Landry et al., 2006). Furthermore, the biofilm life cycle of $P$. aeruginosa has been shown to be dependent on a filamentous prophage (Rice et al., 2009), and the extracellular matrix plays a key role in the post-attachment phase of biofilm formation. Alginate overproduction by mucoid $P$. aeruginosa (Davies \& Geesey, 1995), as well as the expression of two other polysaccharide loci, pel and psl (Friedman \& Kolter, 2004a, b; Ma et al., 2006), and the presence of extracellular DNA (Allesen-Holm et al., 2006; Whitchurch et al., 2002b), contribute to the formation of structured biofilms.

Furthermore, many different cell density-dependent, quorum sensing-regulated functions affect biofilm development (Kirisits \& Parsek, 2006). Therefore, it seems that in addition to cell density, various environmental conditions influence these quorum sensing-controlled functions (Shrout et al., 2006). For example, the induction of Pseudomonas aeruginosa quinolone signal (PQS) signalling, which positively influences $P$. aeruginosa biofilm formation (Diggle et al., 2003; Yang et al., 2007), is dependent on the availability of extracellular iron and phosphate (Jensen et al., 2006).

In this study, we report the screening of the non-redundant set of transposon mutants derived from PA14 (PA14NR) (Liberati et al., 2006) for the ability of the mutants to produce complex biofilms in microtitre plates. This imagebased screen, which was carried out by the use of an automated confocal laser scanning microscope, led to the identification of a large collection of novel mutants affected in biofilm formation. Overall, this result expands our understanding of the genetic factors that control biofilm formation and possibly increases the range of targets for potential therapeutic intervention.

\section{METHODS}

Bacterial strains. The non-redundant set of transposon mutants derived from PA14 (PA14NR) described by Liberati et al. (2006) was used to screen a comprehensive set of mutants for their biofilmforming capability. This library was constructed using a marinerbased transposon containing the resistance cassette aacC1, which confers resistance to gentamicin.
Biofilm growth assays. To assess the capability of the transposon mutants of the Harvard PA14 library to produce biofilms, $5 \mu \mathrm{l}$ samples of a thawed mutant stock were transferred into $150 \mu$ LuriaBertani (LB) broth within a 96-well plate. The preculture plate was covered with an air-permeable BREATHsealcover foil (Greiner BioOne) and incubated at $37{ }^{\circ} \mathrm{C}$ on a shaking unit. After $4 \mathrm{~h}, 5 \mu \mathrm{l}$ of the precultures of each well/mutant were transferred to $100 \mu \mathrm{l} \mathrm{LB}$ medium within a sterile half-area 96-well $\mu$ Clear microplate (Greiner Bio-One). The plates were sealed with a new air-permeable cover foil and placed in an incubator with a humid atmosphere. The image-based screen was carried out after $70 \mathrm{~h}$ of incubation. The incubation was paused after $24 \mathrm{~h}$ in order to stain the bacteria with the LIVE/DEAD BacLight Bacterial Viability kit (Molecular Probes/ Invitrogen). A $50 \mu$ volume of diluted staining solution was added to each well, resulting in a final concentration of $1.4 \mu \mathrm{M}$ Syto 9 and $8.3 \mu \mathrm{M}$ propidium iodide (PI). In each plate, internal controls, representing the PA14 wild-type, and reduced and enhanced biofilm producers, were added (the last control was only included in roughly half of the plates). The PA14 NR pqsA transposon mutant served as a control for poor biofilm formation, whereas an $f \lg F$ mutant derived from the PA14 wild-type served as the control for enhanced biofilm formation.

Automated confocal microscopy. The image-based screen was carried out using the Opera system (Perkin-Elmer-Cellular Technologies), a fully automated confocal microscope suited for the analysis of samples grown in microtitre plates. This system allowed automatic selection of the experimental parameters for image acquisition, which could be applied over the entire screen in order to minimize plate-to-plate variability.

Two positions in each well were chosen to acquire $z$-stacks of the biofilms of a size of $\sim 398 \mu \mathrm{m} \times 300 \mu \mathrm{m}$. Since the biofilms were grown on a polystyrene foil, which may sag slightly in the centre between the edge fixing points, the $z$-stacks were positioned in the centre of each well. A total biofilm height of $30 \mu \mathrm{m}$ was imaged at 11 focal planes starting from the bottom of the plate (position 0), with an inter-plane distance $(z$-step size) of $3 \mu \mathrm{m}$, using a $\times 20 / 0.4$ numerical aperture (NA) air objective. Syto9 was excited with a $488 \mathrm{~nm}$ laser and detected with a 540/75 nm bandpass (BP) emission filter, whereas PI was excited at $561 \mathrm{~nm}$ and detected with a 600/ $40 \mathrm{~nm}$ BP emission filter. Moreover, we selected a dual consecutive exposure mode to prevent optical cross-talk.

Time-series experiments of the internal controls as well as follow-up verification experiments were performed with an Olympus Fluoview 1000 confocal laser scanning microscope equipped with a $\times 20 / 0.75$ NA air objective. The $z$-step size was $2 \mu \mathrm{m}$.

Data analysis. Image stacks obtained by fluorescence microscopy were converted to single '.bmp' files with the software Matlab (version 7.5.0.342, The Mathworks) and further processed to obtain thresholded binary images using the Otsu thresholding algorithm. Afterwards, stacks for each position and dye were reassembled with the software Auto PHLIP-ML (v1.0.0) (Merod et al., 2007), now containing thresholded binary images instead of raw images. Analysis of the different stacks was performed with the Matlab-implemented tool PHLIP (Mueller et al., 2006), producing the following descriptive parameters for the physical properties of the biofilms: biovolume, substratum coverage, area-to-volume ratio, spatial spreading (horizontal, vertical and total), mean thickness and roughness. For the eight parameters extracted from both the Syto9 and the PI image stack, a mean value of the two stack positions was calculated for each mutant. These multidimensional data were visualized within a $2 \mathrm{D}$ projection with a dimensionality reduction method based on singular value decomposition. The dataset was furthermore the basis to distinguish the different biofilm phenotypes of the mutants and to 
categorize them into wild-type-like (normal biofilm), poor (reduced biofilm) and thick (enhanced biofilm) biofilm producer via cluster analysis ( $k$-means clustering) performed with the software Rapidminer [version 4.3 (http://rapidminer.com)]. Although the use of a $\times 20 / 0.4$ NA air objective does not allow the discrimination of live and dead bacteria at the single-cell level, we monitored both red and green fluorescence, because some biofilms exhibited very characteristic staining patterns, thus facilitating the differentiation of biofilm production in the various mutants. $3 \mathrm{D}$ visualization of the biofilm was realized with the software IMARIS x64 (version 5.7.2, Bitplane).

\section{RESULTS AND DISCUSSION}

\section{Global screen to identify genetic determinants of biofilms}

The aim of this study was to identify $P$. aeruginosa PA14 mutants exhibiting an altered biofilm phenotype in a global screen. While multiple genetic screens for modified biofilm formation have been performed in a broad range of bacterial pathogens, including $P$. aeruginosa (EspinosaUrgel et al., 2000; Heilmann et al., 1996; Loo et al., 2000; O’Toole \& Kolter, 1998a, b; Pratt \& Kolter, 1998; Recht \& Kolter, 2001; Tu Quoc et al., 2007; Watnick \& Kolter, 1999), this is believed to be the first report of a microscopic screen for mutants forming altered biofilms.

An absolute pre-requirement for the applicability of a 96-well plate-based optical method to serve as a reproducible and robust screen for mutants forming altered biofilms is that multiple equivalent biofilms are formed in the individual wells of the microtitre plate. Fig. 1 shows 3D biofilm images and the c.f.u. counts of PA14 grown in eight individual wells of one 96-well plate as well as the processed data (total biovolume) of the replicates. No major differences were noted when comparing images, c.f.u. counts or processed data in the replicates of the PA14 reference strain.

Next, we assessed the robustness and discriminating power of our image-based method to determine the ability of mutants to form biofilms at the bottom of 96-well plates using a confocal laser scanning microscope after live and dead staining of the cells. Three PA14 NR mutants ( $p q s A$, $p q s E$ and $p q s L$ ) harbouring a transposon insertion within genes involved in alkyl-quinolone signalling, [described elsewhere as playing a role in biofilm formation (Diggle et al., 2003)], and an flgF mutant derived from the PA14 wild-type, were grown in at least 11 replicates in one 96well plate for $48 \mathrm{~h}$. Images of the biofilm stacks were acquired and physical parameters (see Methods) were determined and used for hierarchical clustering. The cluster tree in Fig. 2(a) demonstrates the successful discrimination of the different mutant biofilms. Representative images of the various biofilm morphologies are shown in Fig. 2(b). The applied optical screen clearly proved to be sufficient to discriminate high ( $f l g F$ mutant) from low ( $p q s A$ mutant) biofilm producers. However, (a)

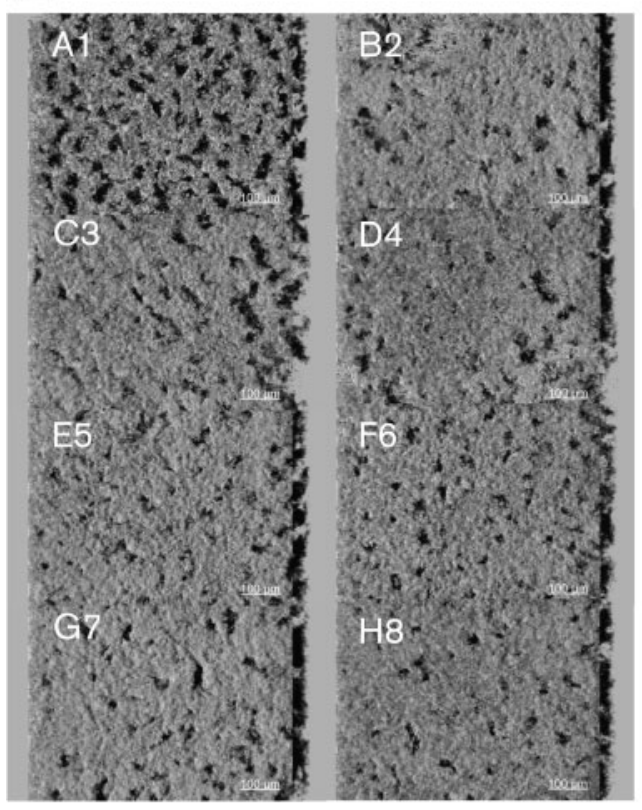

(b)

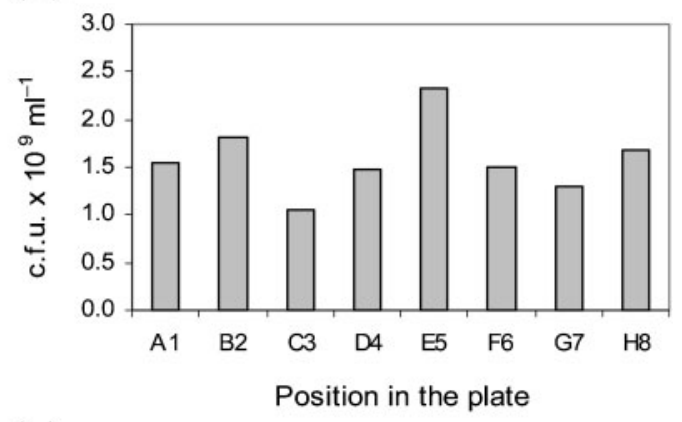

(c)

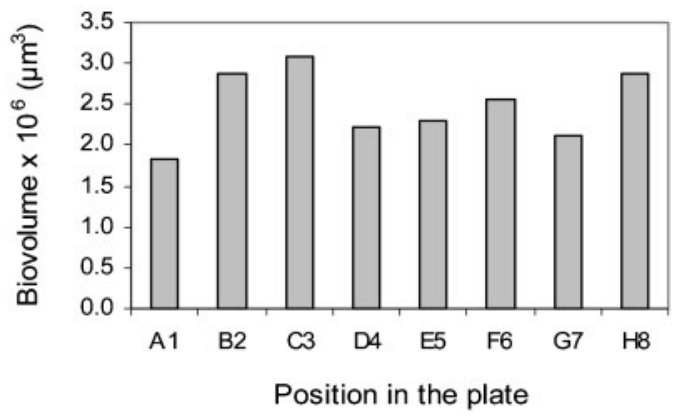

Fig. 1. (a) 3D visualizations, (b) c.f.u. counts and (c) total biovolume of PA14 wild-type biofilms at chosen positions of a 96-well plate. Bars, $100 \mu \mathrm{m}$. 
(a)

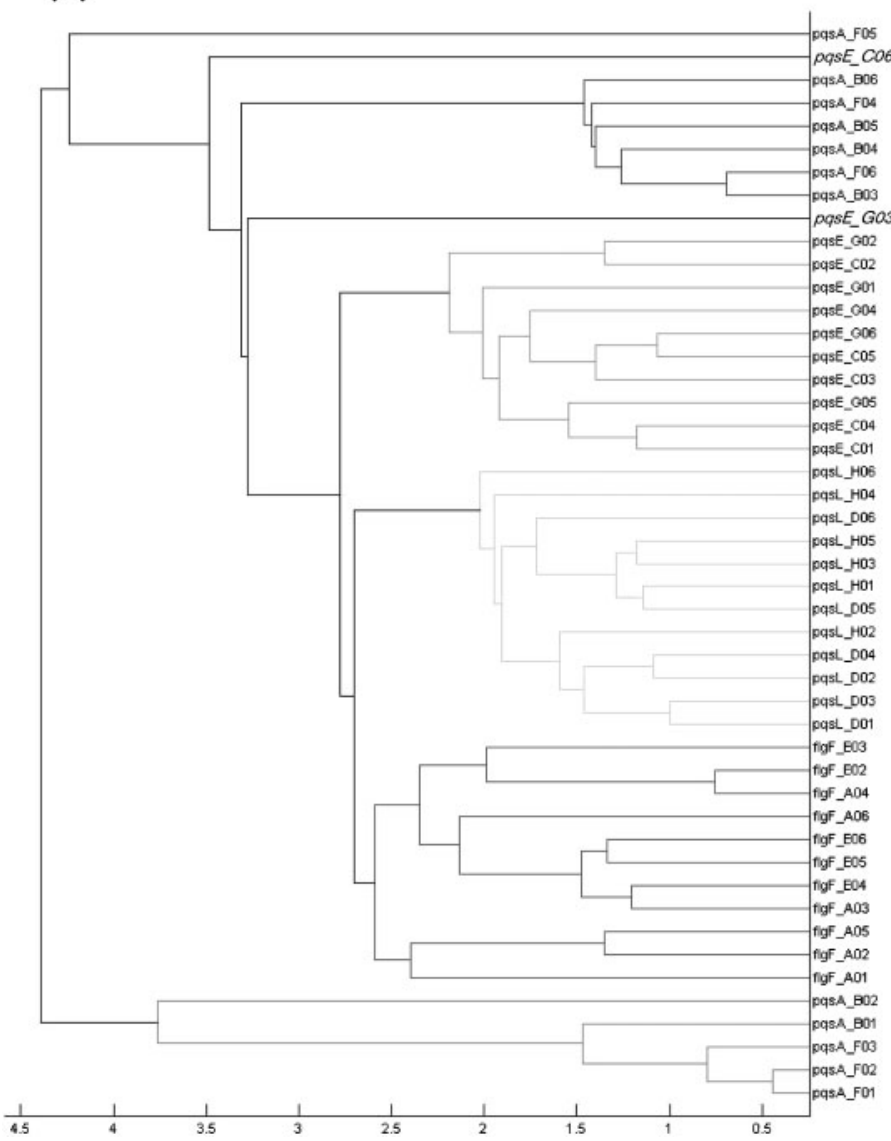

(b)
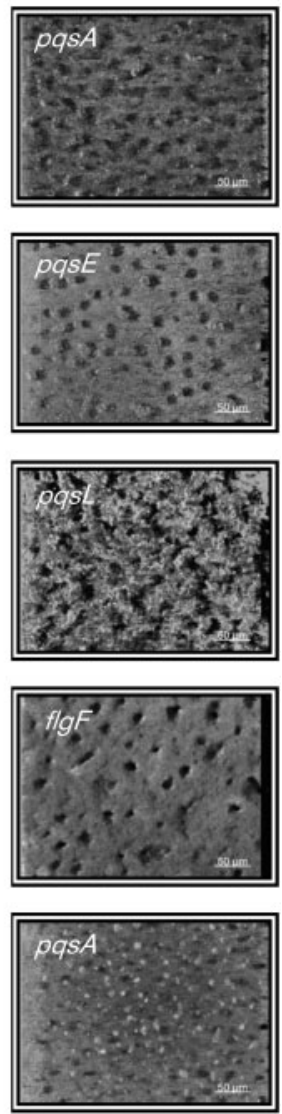

Fig. 2. Hierarchical clustering of the four PA14NR mutants $p q s A, p q s E$, pqs $L$ and $f / g F(a)$, and a representative 3D image of each mutant (b). The two pqsE mutants which were found outside of the pqs $E$ cluster are shown in italic type. Bars, $50 \mu \mathrm{m}$.

whereas all replicates of the high biofilm-producing and wild-type-like mutants ( $p q s L$ mutant) were found within the same cluster, there were two $p q s E$ mutant and one $p q s A$ mutant biofilms which did not cluster with the other replicates of the respective mutant group. This intra-strain variability can often be explained by image-conversion errors, e.g. during the Otsu-thresholding of overexposured images or of low-contrast images in general, which has a stronger impact on low-biofilm producers. Nevertheless, the intra-strain variability was much smaller than the interstrain variability between high and low biofilm-producing strains, as Fig. 2(a) clearly demonstrates.

We next monitored the biofilm life cycle of the $p q s A$ mutant, which we selected as a control strain for poor biofilm formation, and of the flgF mutant, selected as a control strain for enhanced biofilm production, in comparison over time with that of the PA14 wild-type. As depicted in Fig. 3, the initial bacterial lawn which covered the bottom of the wells after $24 \mathrm{~h}$ of incubation became more and more structured and accentuated over time, whereas after $96 \mathrm{~h}$ bacteria began to disperse. Because the characteristics of the wild-type, poor and enhanced biofilms were most pronounced at $72 \mathrm{~h}$, we decided to screen the PA14NR library for altered biofilm formation at this time point of incubation.

In order to ascertain the sensitivity and specificity of the screening method and to further address the inter-plate variability, PA14 wild-type and poor-biofilm controls were included in each of the 96-well screening plates, whereas the enhanced-biofilm control was added to roughly half of the microtitre plates. A $2 \mathrm{D}$ projection based on singular value decomposition of the 16 discriminative parameters of all 5833 mutants in addition to 536 internal controls (180 wild-type, $268 p q s A$ and 88 flgF mutants) is shown in Fig. 4. The boundaries of the three groups, including mutants with reduced, normal and enhanced biofilms, were defined following $k$-means clustering, and were set very conservatively in order to strictly minimize the identification of false-positive mutants with reduced or enhanced biofilm production. However, this resulted in higher rates of falsenegative mutants, probably leading to a strong underestimation of the number of factors important for biofilm 
(a)
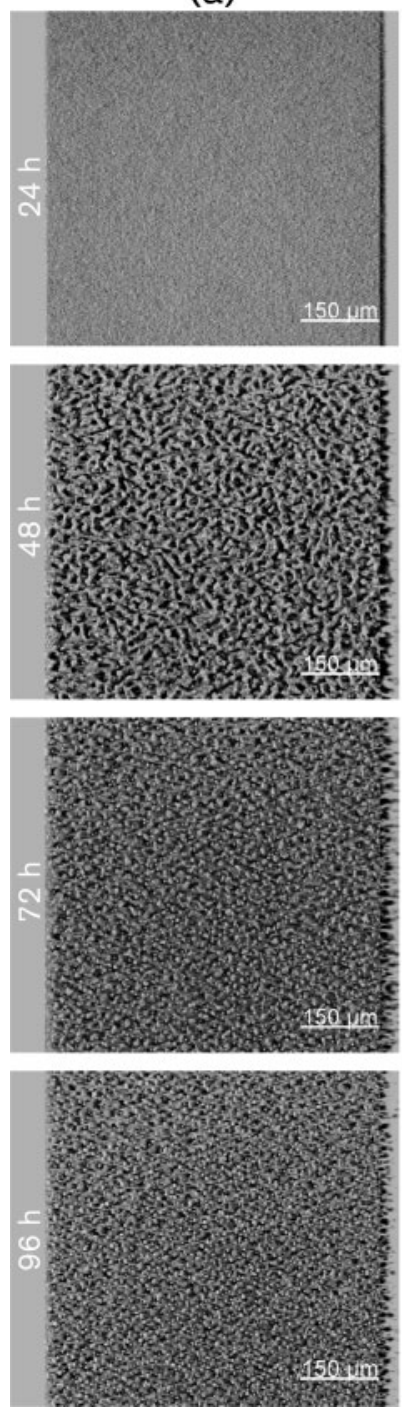

(b)
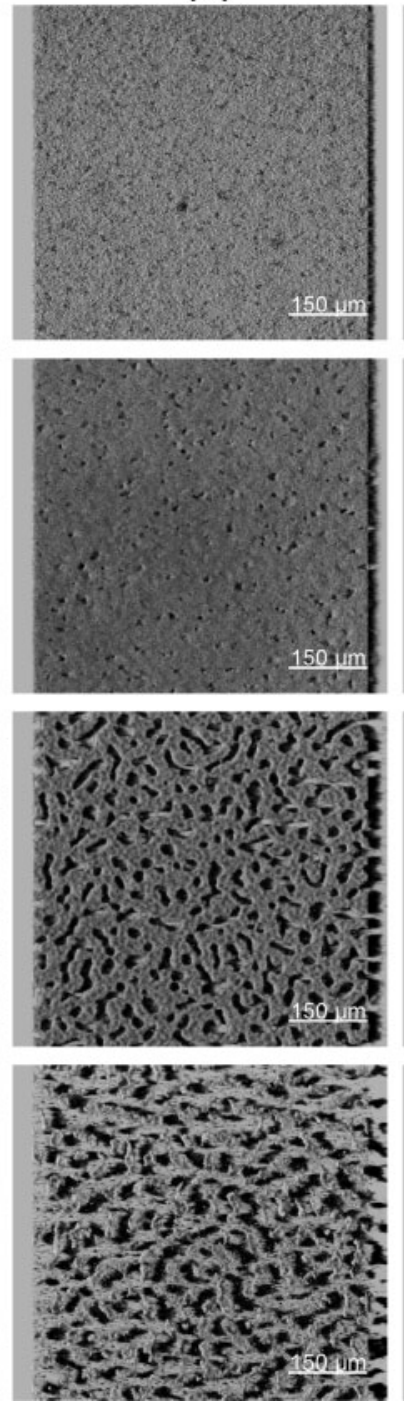

(c)
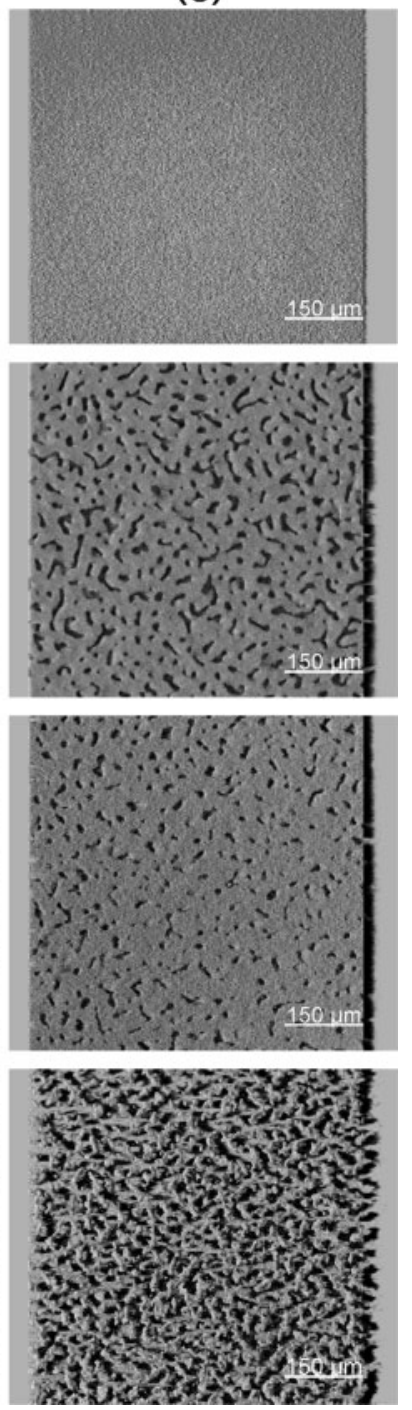

Fig. 3. $3 D$ visualization of representative PA14 biofilms of the internal controls: (a) poor biofilm (pqs $A$ mutant), (b) normal biofilm (wild-type) and (c) enhanced biofilm (flgF mutant) after 24, 48, 72 and $96 \mathrm{~h}$ of incubation. Bars, $150 \mu \mathrm{m}$.

formation, a fact which might explain why our screen missed some known biofilm-forming factors.

In our genome-wide screen, the conservative grouping classified none of the poor-biofilm controls in the enhanced-biofilm group and vice versa. In addition, none of the 35 mutants with the same mutant ID that appear twice in the library was found in both the high- and the poor-biofilm clusters. Nevertheless, we found that inactivation of 12 out of 1025 genes, where more than one transposon was inserted (at a different position within the gene), resulted in either an enhanced- or a poor-biofilm phenotype. This finding probably reflects the limitation of the screen, where overexposure, cross-contamination, incomplete dye penetration, foreign particles in the field, image-processing errors and false assignment of the PA14NR mutants might lead to misinterpretations.

In Supplementary Table S1, the PA14 mutant subgroup exhibiting poor biofilms as compared with the wild-type is listed. This subgroup comprises $7 \%$ of all tested mutants, $81.7 \%$ of all poor-biofilm controls, only $11.6 \%$ of the PA14 wild-type and none of the PA14 enhanced-biofilm controls. Supplementary Table S2, on the other hand, lists the PA14 mutant subgroup which comprises roughly $5 \%$ of all mutants exhibiting an enhanced-biofilm phenotype. This mutant subgroup included $50 \%$ of all enhancedbiofilm controls, only $8.9 \%$ of the PA14 wild-type and none of the PA14 poor-biofilm controls. The relatively high rate of misinterpretation clearly demonstrates the limita- 


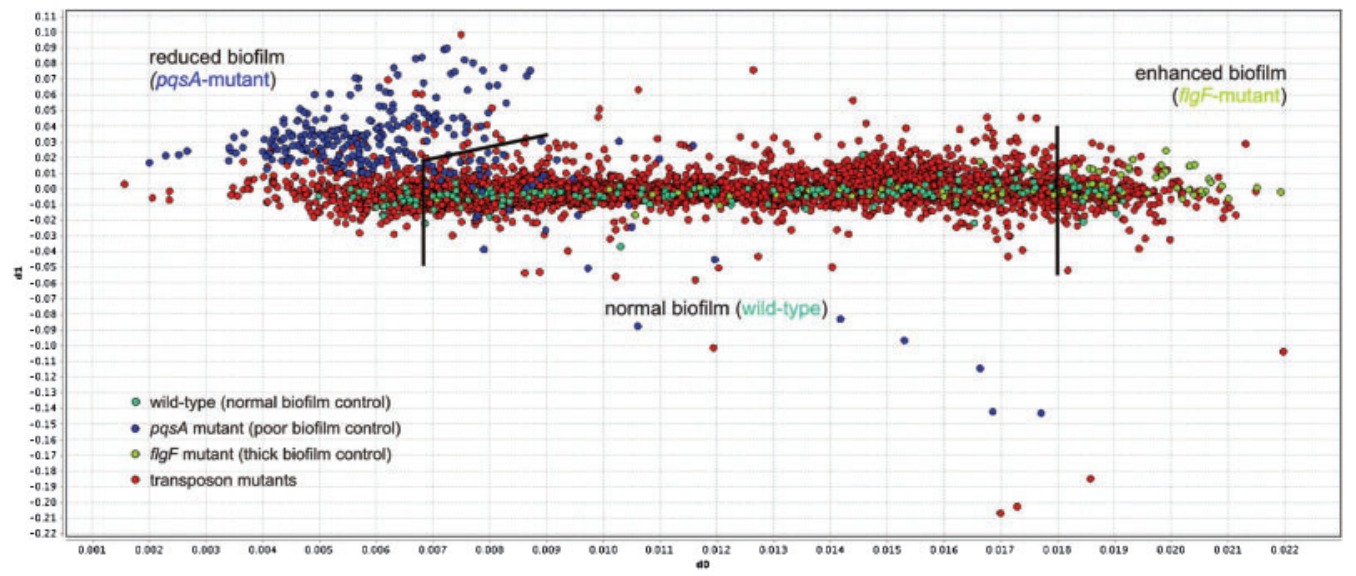

Fig. 4. $2 \mathrm{D}$ projection of the distribution of the whole transposon mutant library. Cluster boundaries are shown by black lines; mutants and internal controls are coloured as indicated.

tion of our screen, especially in the detection of enhanced biofilm-producing strains, where the conservative grouping, chosen to assure high specificity, led to very low sensitivity (only $50 \%$ ).

In order to estimate the effect of growth on biofilm formation we searched for the $5 \%$ of the mutants which showed the lowest growth in planktonic cultures (as identified in the study of Dötsch et al. (2009) and determined their distribution among the poor- and enhanced-biofilm categories, respectively. We found $4.2 \%$ of the slow-growing mutants within the list of enhanced biofilm-forming mutants and $10.9 \%$ in the poor biofilmforming group. This indicates that slow-growing mutants tend to be, but are not necessarily, poor biofilm producers.

Interestingly, among the mutants with defective biofilm formation, $64.7 \%$ of the affected genes encoded hypothetical proteins, whereas $69.1 \%$ of the enhanced biofilmforming mutants encoded hypothetical proteins. These data indicate that there are many yet to be discovered regulatory cascades required for biofilm establishment.

It should be noted that the assignment of a single PA14NR mutant to the wild-type, poor- or enhanced-biofilm group should be interpreted with care. The assignment of the PA14NR mutants might be erroneous, false-positive and especially false-negative mutants cannot be excluded (see above), and the screening of the 5833 mutants was only done once, and although the biofilm growth conditions within the microtitre plates were highly standardized, deviations in, e.g. inoculum size, microscope settings and staining procedures, might lead to misinterpretations.

Thus, in consideration of a potential miscategorization of single PA14NR mutants, we will concentrate in this manuscript on the discussion of sets of functionally related mutants that were found to be affected in biofilm formation.

\section{Rare detection of previously identified genetic determinants of biofilm formation}

Our approach uncovered 394 (Supplementary Table S1) and 285 (Supplementary Table S2) genetic determinants that contribute to the biofilm phenotype of $P$. aeruginosa PA14. Only very few of these genes have previously been detected in global screens for factors participating in biofilm formation in $P$. aeruginosa or other bacterial pathogens (Espinosa-Urgel et al., 2000; Heilmann et al., 1996; Loo et al., 2000; O’Toole \& Kolter, 1998a, b; Pratt \& Kolter, 1998; Recht \& Kolter, 2001; Tu Quoc et al., 2007; Watnick \& Kolter, 1999). Furthermore, we observed a discrepancy not only in the identity of the contributing factors but also in the number of mutants detected. The use of an optical detection method in this study identified a proportion of poor biofilm producers that exceeded by far those of most previous genetic screens. The main reason seems to be that, whereas previous screens focused on the identification of genes involved in the initial steps of adhesion, we analysed mature biofilms.

The first global genetic screen for mutants that form altered biofilms in P. aeruginosa was performed by O'Toole \& Kolter (1998a). Their screen uncovered 15 surface attachment deficient (sad) mutants (Kukavica-Ibrulj et al., 2008); however, only one of these mutants $(s a d B)$ was detected within our screen. Instead, we observed that sad mutants with defects in swimming motility produced more dense biofilms than the wild-type. This apparent inconsistency strongly supports the earlier finding that the requirement for flagelladriven motility for the establishment of biofilms varies depending on environmental conditions (Klausen et al., 2003). Whereas O'Toole and Kolter used minimal medium to cultivate pseudomonads in microtitre plates at the air-liquid interface (O'Toole \& Kolter, 1998a, b), we used a rich medium (LB broth) to promote biofilm formation at the bottom of the microtitre plate under low-oxygen conditions. 
Since 1998, various additional factors have been associated with the biofilm lifestyle in $P$. aeruginosa, including GacA (Parkins et al., 2001), CupA (Vallet et al., 2001), LasI (Davies et al., 1998), the catabolite repression control protein Crc (O'Toole et al., 2000), PslA (Matsukawa \& Greenberg, 2004) and AlgR (Whitchurch et al., 2002a). With the expection of a cupA3 and a pslG PA14NR mutant, none of the other mutants was identified in our screen.

Furthermore, it has become increasingly apparent that the intracellular signalling molecule cyclic di-GMP (c-diGMP) is involved in the regulation of the transition from the planktonic to the surface-attached biofilm mode of growth in many Gram-negative bacterial pathogens (Jenal \& Malone, 2006; Römling \& Amikam, 2006). c-di-GMP is synthesized by a family of enzymes called diguanylate cyclases (DGCs) and degraded by c-di-GMP phosphodiesterases (PDEs). Although nine $P$. aeruginosa mutants in genes encoding both DGCs and proteins which possess both GGDEF and EAL domains have been reported to exhibit abolished or reduced biofilm formation capability, and six mutants enhanced biofilm formation capabilities (Kulasakara et al., 2006), we only identified two mutants with a transposon insertion within a gene encoding a DGC as forming reduced biofilms (PA0290 and PA5487). Interestingly, a transcriptional analysis of biofilm-grown $P$. aeruginosa $\mathrm{PAO} 1$ has indicated expression of PA0290 to be significantly enhanced (Waite et al., 2005).

\section{Genetic determinants promoting survival under microaerophilic conditions are essential for efficient $\boldsymbol{P}$. aeruginosa biofilm formation}

Among the 394 biofilm-deficient mutants, we identified the two main components of the rhl quorum-sensing system, $r h l R$ and $r h l I$. The $r h l$ system is known to be highly expressed under biofilm (Singh et al., 2000) and microaerophilic conditions (Alvarez-Ortega \& Harwood, 2007), and P. aeruginosa has been shown to form robust anaerobic biofilms, the viability of which requires the rhl quorumsensing system (Yoon et al., 2002). Rhl mutants exhibit high levels of toxic NO, which seems to account for premature cell death (Yoon et al., 2002). We also identified an oprF mutant as forming poor biofilms in our screening assay. OprF has been shown to be highly expressed in clinical CF $P$. aeruginosa isolates (Sriramulu et al., 2005). A proteomic analysis has revealed a marked upregulation of OprF under anaerobic conditions (Platt et al., 2008), and OprF has been suggested to be important for $\mathrm{NO}$ detoxification (Yoon et al., 2002) and thus for the establishment of biofilms.

We also identified two poor-biofilm mutants affected in genes with predicted terminal oxidase activity. One of them has been described to be preferentially expressed in the stationary phase of growth (CoxA) (Schuster et al., 2004), and the other, a cyanide-insensitive $b d$-type oxidase (cio), has been predicted to have a high affinity for oxygen and shown to contribute together with the $c b b_{3}-1$ and $c b b_{3}-2$ oxidases to sustaining respiration when oxygen levels are low (Alvarez-Ortega \& Harwood, 2007). We did not identify mutants of the $c b b_{3}$ oxidases to exhibit a reducedbiofilm phenotype. However, we observed a clear defect in biofilm formation in the cioB mutant, although a cioA mutant has been described to exhibit biofilm formation capabilities similar to those of the wild-type when grown in a flow chamber (Alvarez-Ortega \& Harwood, 2007). Interestingly, those authors showed that expression of the hydrogen cyanide synthase genes was activated about fivefold in a transcriptome analysis under microaerophilic conditions and suggested that the cyanide-insensitive oxidase exerts the additional role of protecting the cells from hydrogen cyanide toxicity during microaerophilic growth. This scenario might be of major importance under our experimental conditions, and might also explain why cultivation of a cioA mutant within the flow chamber does not show a biofilm-defective phenotype, because HCN is more efficiently eliminated.

Furthermore, it is interesting to note that we identified additional poor-biofilm mutants that were affected in genes described to be pronouncedly upregulated under microaerophilic/anaerobic growth conditions, including $h u t U$, encoding a urocanase (Alvarez-Ortega \& Harwood, 2007), PA4142, a conserved hypothetical gene within the same operon that encodes a predicted $4 \mathrm{Fe}-4 \mathrm{~S}$ ferredoxin transmembrane protein and a homologue of the catalytic subunit of $c b b_{3}$ oxidase (CcoN) (Alvarez-Ortega \& Harwood, 2007), PA5481 (Alvarez-Ortega \& Harwood, 2007), azu (Vijgenboom et al., 1997), nirE, nirJ, nosL and ptxR (Gaines et al., 2007).

\section{Arginine metabolism is essential for $P$. aeruginosa biofilms}

When oxygen availability becomes limited, a central metabolic process to generate ATP in $P$. aeruginosa is arginine fermentation via the arginine deaminase (ADI) pathway (Lu et al., 2004). Although we did not find mutants affected in the ADI pathway to exhibit a reduced biofilm formation phenotype, it is interesting to note that we identified several mutants with insertions within genes involved in the pyrimidine nucleotide biosynthetic pathway ( $\operatorname{pyr} B C, \operatorname{car} A B)$. The first step in this pathway is the formation of carbamoyl phosphate by carbamoyl-phosphate synthase, which is encoded by the $c a r A B$ genes. Carbamoyl phosphate is also required for the biosynthesis of arginine from ornithine via the arginine metabolic pathway, which involves $\operatorname{argFGH}$ (Lu et al., 2004). We identified not only mutants affected in the pyrimidine nucleotide biosynthetic pathway but also an $\arg G$ and $\operatorname{argH}$ mutant affected in the formation of arginine from carbamoyl phosphate and ornithine, and two mutants ( $g a b T$ and $a r u G$ ) affected in arginine and ornithine degradation, respectively. It therefore seems that $P$. aeruginosa PA14 requires a functional arginine metabolism in order to establish robust biofilms; accordingly, arginine probably serves as a source of carbon, energy and nitrogen. 
Furthermore, we identified a mutant with an insertion within the cbrA gene which exhibited a reduced-biofilm phenotype. It has been shown that $\mathrm{CbrAB}$ is a twocomponent system essential for the expression of ArgRdependent pathways (Nishijyo et al., 2001). Since ArgR is the major regulator of arginine and glutamine metabolism (Lu et al., 2004), this finding underscores the importance of arginine metabolism for the establishment of $P$. aeruginosa biofilms.

Most interestingly, in Vibrio parahaemolyticus (EnosBerlage et al., 2005), a carA transposon mutant has been shown to form only thin pellicles at the air-medium interface, and a comparison of the previously published transcriptome profiles of S. aureus (Beenken et al., 2004), Legionella pneumophila (Hindré et al., 2008) and Escherichia coli (Schembri et al., 2003) revealed a common set of highly expressed genes under biofilm conditions that are involved in the pyrimidine nucleotide biosynthetic pathway as well as arginine metabolism. PyrRPBC, carAB, pyrFE, $\arg D A B C$ and $\arg G H$ are highly expressed in $S$. aureus biofilms (Beenken et al., 2004); pyrGH, carA and $\operatorname{argD}$ in L. pneumophila biofilms (Hindré et al., 2008); and artJ, pyrB, pyrI, gltB, glnA, gltD, $\arg C$ and $\operatorname{argF}$ in E. coli biofilms (Schembri et al., 2003), indicating that a functional arginine metabolism is essential for biofilm formation in various bacterial species. Arginine has been shown to enhance the formation of $P$. aeruginosa biofilms (Caiazza \& O'Toole, 2004). With the aim of evaluating the effect of arginine on biofilm formation under our experimental conditions, we exogenously added arginine to the PA14 biofilm cultures and clearly observed enhanced biofilm formation at arginine concentrations of $0.4 \%$ $(w / v)$ in the wild-type and the $\arg G$ and $\arg H$ mutants (data not shown).

\section{pH homeostasis is essential for $\boldsymbol{P}$. aeruginosa biofilms}

One of the most extensively differentially regulated genes, which was identified in the first screen of the global $P$. aeruginosa gene expression profile under biofilm growth conditions, is ureB, encoding a urease (Whiteley et al., 2001). This enzyme has been described to be markedly upregulated in Streptococcus salivarius (Li et al., 2000) and S. aureus (Beenken et al., 2004) biofilms, and it has been speculated that it is involved in the maintenance of $\mathrm{pH}$ homeostasis in biofilm cultures. Ureases hydrolyse urea to two molecules of ammonia and one of carbon dioxide, which results in efficient alkalinization of the environment as an alternative to the generation of ammonia from the ADI pathway. Our findings indicate that $\mathrm{pH}$ homeostasis is crucial to PA14 biofilm formation in our experimental setting. We found not only various mutants affected in arginine metabolism (see above) that exhibited poor biofilms but also a ureB mutant, which produced low levels of biofilms. Furthermore we identified a $k d p D$ mutant as developing poor biofilms. In addition to the production of ammonia, cation transport ATPases, such as the high-affinity $\mathrm{K}^{+}$-specific transport system encoded by the $k d p$ operon, can also contribute to $\mathrm{pH}$ homeostasis through the exchange of $\mathrm{K}^{+}$for $\mathrm{H}^{+}$. In E. coli, $\mathrm{KdpD}$ and $\mathrm{KdpE}$, proteins that control expression of the $k d p F A B C$ operon, are members of the class of sensor kinase/response regulator proteins (Voelkner et al., 1993).

In order to evaluate the impact of the $\mathrm{pH}$ on biofilm development under our experimental conditions, we grew PA14 wild-type biofilms in HEPES-buffered LB medium at various $\mathrm{pH}$ values. As compared with non-buffered controls, biofilm establishment was seriously affected under buffered LB medium conditions (data not shown).

\section{Genes essential for the repair and maintenance of DNA are required for efficient biofilm formation}

The screen for biofilm-deficient mutants uncovered the importance of six genes involved in DNA repair mechanisms ( $r e c A, r e c B, r e c G, r e c J$, $r e c N$ and $u v r D$ ). RecA has very recently been described to affect biofilm development in Streptococcus mutans (Inagaki et al., 2009). Most interestingly, in $P$. aeruginosa it has been demonstrated that double-stranded DNA break repair is required for biofilmmediated diversity and that an endogenous oxidative stress triggers a DNA repair mechanism that in turn generates genetic variants with a wide range of fitness characteristics (Boles et al., 2004; Boles \& Singh, 2008). It therefore seems that mutants with defects in the generation of self-induced diversity do not withstand the intrinsic and extrinsic stresses that act upon biofilm bacteria in our 96-well format system.

\section{Interbacterial signalling contributes to biofilm formation}

In addition to the mutants within the rhl quorum-sensing system, we also identified a mexH and mexI mutant as forming deficient biofilms. The MexH protein was recently described to be upregulated at the protein level during biofilm development and to play a critical role in biofilm maturation (Southey-Pillig et al., 2005). This might be due to the influence of the MexGHI-OpmD pump on interbacterial communication, since a recent report shows that mutation of genes encoding the pump results in the inability to produce $\mathrm{N}$-(3-oxododecanoyl)-L-homoserine lactone (3-oxo-c12-hsl) and alkyl-quinolones (Aendekerk et al., 2005). Our screen identified $p q s A$ and $p q s C$ mutants as poor biofilm producers, which is in agreement with the findings of Diggle et al. (2003), who showed that diminished alkyl-quinolone production results in low biofilm production. Unexpectedly, the $p q s D$ mutant was categorized within the group of enhanced-biofilm builders; however, this mutant produced alkyl-quinolones at levels comparable with those of the PA14 wild-type, indicating a misassignment. Interestingly, mutations within the pyrimidine pathway (carB, pyrB and pyrD) have previously 
been described to exhibit a reduced production of the alkyl-quinolones, which could be complemented in both growth and alkyl-quinolone production by the exogenous addition of UMP (Bredenbruch et al., 2005). Although the low alkyl-quinolone production may contribute to the low biofilm production of the pyrimidine mutants, the finding that enzymes of the pyrimidine biosynthetic pathway are upregulated under biofilm growth conditions in various bacterial pathogens (see above) suggests that other mechanisms, such as the requirement for arginine, also contribute to the poor biofilm formation.

\section{Conclusions}

The study of biofilm development in various bacterial pathogens by the use of genetic screens and the application of transcriptional profiling under biofilm growth conditions have uncovered several complex regulatory systems required for surface-attached growth of the respective pathogens and revealed some general strategies for the initiation of biofilms (Beloin \& Ghigo, 2005; Kuchma \& O’Toole, 2000; Lazazzera, 2005). In this study we show the power of combining a genetic screen with microscopic analysis to uncover several novel genetic determinants of $P$. aeruginosa biofilm formation and maintenance. Although the relatively high amount of false positives and false negatives is clearly a drawback of the automated technique used in this study, it seems that some of the identified genetic determinants of biofilm formation, such as the requirement for arginine metabolism, $\mathrm{pH}$ homeostasis and survival under microaerophilic growth conditions, are common themes for the establishment and maintenance of biofilms that have also emerged from earlier analyses of global biofilm transcriptional profiles in various pathogens.

\section{ACKNOWLEDGEMENTS}

Financial support from the Mukoviszidose e.V., the HelmholtzGemeinschaft and the Bundesministerium für Bildung und Forschung (BMBF) is gratefully acknowledged. M. M. was supported by the International Research Training Group 1273 funded by the German Research Foundation (DFG). A.D. is a recipient of a predoctoral stipend provided by the DFG-sponsored International Research Training Group 'Pseudomonas: Pathogenicity and Biotechnology'.

\section{REFERENCES}

Aendekerk, S., Diggle, S. P., Song, Z., Hoiby, N., Cornelis, P., Williams, P. \& Camara, M. (2005). The MexGHI-OpmD multidrug efflux pump controls growth, antibiotic susceptibility and virulence in Pseudomonas aeruginosa via 4-quinolone-dependent cell-to-cell communication. Microbiology 151, 1113-1125.

Allesen-Holm, M., Barken, K. B., Yang, L., Klausen, M., Webb, J. S., Kjelleberg, S., Molin, S., Givskov, M. \& Tolker-Nielsen, T. (2006). A characterization of DNA release in Pseudomonas aeruginosa cultures and biofilms. Mol Microbiol 59, 1114-1128.

Alvarez-Ortega, C. \& Harwood, C. S. (2007). Responses of Pseudomonas aeruginosa to low oxygen indicate that growth in the cystic fibrosis lung is by aerobic respiration. Mol Microbiol 65, 153165.

Beenken, K. E., Dunman, P. M., McAleese, F., Macapagal, D., Murphy, E., Projan, S. J., Blevins, J. S. \& Schmeltzer, M. S. (2004). Global gene expression in Staphylococcus aureus biofilms. J Bacteriol 186, 4665-4684.

Beloin, C. \& Ghigo, J. M. (2005). Finding gene-expression patterns in bacterial biofilms. Trends Microbiol 13, 16-19.

Boles, B. R. \& Singh, P. K. (2008). Endogenous oxidative stress produces diversity and adaptability in biofilm communities. Proc Natl Acad Sci U S A 105, 12503-12508.

Boles, B. R., Thoendel, M. \& Singh, P. K. (2004). Self-generated diversity produces "insurance effects" in biofilm communities. Proc Natl Acad Sci U S A 101, 16630-16635.

Bredenbruch, F., Nimtz, M., Wray, V., Morr, M., Müller, R. \& Häussler, S. (2005). Biosynthetic pathway of Pseudomonas aeruginosa 4-hydroxy-2alkylquinolines. J Bacteriol 187, 3630-3635.

Caiazza, N. C. \& O'Toole, G. A. (2004). SadB is required for the transition from reversible to irreversible attachment during biofilm formation by Pseudomonas aeruginosa PA14. J Bacteriol 186, 44764485.

Costerton, J. W., Lewandowski, Z., Caldwell, D. E., Korber, D. R. \& Lappin-Scott, H. M. (1995). Microbial biofilms. Annu Rev Microbiol 49, 711-745.

Davies, D. G. \& Geesey, G. G. (1995). Regulation of the alginate biosynthesis gene algC in Pseudomonas aeruginosa during biofilm development in continuous culture. Appl Environ Microbiol 61, 860-867.

Davies, D. G., Parsek, M. R., Pearson, J. P., Iglewski, B. H., Costerton, J. W. \& Greenberg, E. P. (1998). The involvement of cell-to-cell signals in the development of a bacterial biofilm. Science 280, 295298.

Diggle, S. P., Winzer, K., Chhabra, S. R., Worrall, K. E., Camara, M. \& Williams, P. (2003). The Pseudomonas aeruginosa quinolone signal molecule overcomes the cell density-dependency of the quorum sensing hierarchy, regulates $r h l$-dependent genes at the onset of stationary phase and can be produced in the absence of LasR. Mol Microbiol 50, 29-43.

Dötsch, A., Becker, T., Pommerenke, C., Magnowska, Z., Jänsch, L. \& Häussler, S. (2009). Genomewide identification of genetic determinants of antimicrobial drug resistance in Pseudomonas aeruginosa. Antimicrob Agents Chemother 53, 2522-2531.

Enos-Berlage, J. L., Guvener, Z. T., Keenan, C. E. \& McCarter, L. L. (2005). Genetic determinants of biofilm development of opaque and translucent Vibrio parahaemolyticus. Mol Microbiol 55, 1160-1182.

Espinosa-Urgel, M., Salido, A. \& Ramos, J. L. (2000). Genetic analysis of functions involved in adhesion of Pseudomonas putida to seeds. J Bacteriol 182, 2363-2369.

Friedman, L. \& Kolter, R. (2004a). Genes involved in matrix formation in Pseudomonas aeruginosa PA14 biofilms. Mol Microbiol 51, 675-690.

Friedman, L. \& Kolter, R. (2004b). Two genetic loci produce distinct carbohydrate-rich structural components of the Pseudomonas aeruginosa biofilm matrix. J Bacteriol 186, 4457-4465.

Gaines, J. M., Carty, N. L., Tiburzi, F., Davinic, M., Visca, P., ColmerHamood, J. A. \& Hamood, A. N. (2007). Regulation of the Pseudomonas aeruginosa toxA, regA and $p t x R$ genes by the ironstarvation sigma factor $\mathrm{PvdS}$ under reduced levels of oxygen. Microbiology 153, 4219-4233.

Gilbert, P. \& McBain, A. J. (2001). Biofilms: their impact on health and their recalcitrance toward biocides. Am J Infect Control 29, 252255. 
Gilbert, P., Maira-Litran, T., McBain, A. J., Rickard, A. H. \& Whyte, F. W. (2002). The physiology and collective recalcitrance of microbial biofilm communities. Adv Microb Physiol 46, 202-256.

Heilmann, C., Gerke, C., Perdreau-Remington, F. \& Gotz, F. (1996). Characterization of Tn917 insertion mutants of Staphylococcus epidermidis affected in biofilm formation. Infect Immun 64, 277-282.

Hindré, T., Brüggemann, H., Buchrieser, C. \& Héchard, Y. (2008). Transcriptional profiling of Legionella pneumophila biofilm cells and the influence of iron on biofilm formation. Microbiology 154, 30-41.

Inagaki, S., Matsumoto-Nakano, M., Fujita, K., Nagayama, K., Funao, J. \& Ooshima, T. (2009). Effects of recombinase A deficiency on biofilm formation by Streptococcus mutans. Oral Microbiol Immunol 24, 104108.

Jenal, U. \& Malone, J. (2006). Mechanisms of cyclic-di-GMP signaling in bacteria. Annu Rev Genet 40, 385-407.

Jensen, V., Löns, D., Zaoui, C., Bredenbruch, F., Meissner, A., Dieterich, G., Münch, R. \& Häussler, S. (2006). RhlR expression in Pseudomonas aeruginosa is modulated by the Pseudomonas quinolone signal via PhoB-dependent and -independent pathways. J Bacteriol 188, 8601-8606.

Kirisits, M. J. \& Parsek, M. R. (2006). Does Pseudomonas aeruginosa use intercellular signalling to build biofilm communities? Cell Microbiol 8, 1841-1849.

Klausen, M., Heydorn, A., Ragas, P., Lambertsen, L., AaesJorgensen, A., Molin, S. \& Tolker-Nielsen, T. (2003). Biofilm formation by Pseudomonas aeruginosa wild type, flagella and type IV pili mutants. Mol Microbiol 48, 1511-1524.

Kuchma, S. L. \& O'Toole, G. A. (2000). Surface-induced and biofilminduced changes in gene expression. Curr Opin Biotechnol 11, 429433.

Kukavica-Ibrulj, I., Bragonzi, A., Paroni, M., Winstanley, C., Sanschagrin, F., O'Toole, G. A. \& Levesque, R. C. (2008). In vivo growth of Pseudomonas aeruginosa strains PAO1 and PA14 and the hypervirulent strain LESB58 in a rat model of chronic lung infection. J Bacteriol 190, 2804-2813.

Kulasakara, H., Lee, V., Brencic, A., Liberati, N., Urbach, J., Miyata, S., Lee, D. G., Neely, A. N., Hyodo, M. \& other authors (2006). Analysis of Pseudomonas aeruginosa diguanylate cyclases and phosphodiesterases reveals a role for bis- $\left(3^{\prime}-5^{\prime}\right)$-cyclic-GMP in virulence. Proc Natl Acad Sci U S A 103, 2839-2844.

Landry, R. M., An, D., Hupp, J. T., Singh, P. K. \& Parsek, M. R. (2006). Mucin-Pseudomonas aeruginosa interactions promote biofilm formation and antibiotic resistance. Mol Microbiol 59, 142-151.

Lazazzera, B. A. (2005). Lessons from DNA microarray analysis: the gene expression profile of biofilms. Curr Opin Microbiol 8, 222-227.

Li, Y. H., Chen, Y. Y. \& Burne, R. A. (2000). Regulation of urease gene expression by Streptococcus salivarius growing in biofilms. Environ Microbiol 2, 169-177.

Liberati, N. T., Urbach, J. M., Miyata, S., Lee, D. G., Drenkard, E., Wu, G., Villanueva, J., Wei, T. \& Ausubel, F. M. (2006). An ordered, nonredundant library of Pseudomonas aeruginosa strain PA14 transposon insertion mutants. Proc Natl Acad Sci U S A 103, 2833-2838.

Loo, C. Y., Corliss, D. A. \& Ganeshkumar, N. (2000). Streptococcus gordonii biofilm formation: identification of genes that code for biofilm phenotypes. J Bacteriol 182, 1374-1382.

Lu, C. D., Yang, Z. \& Li, W. (2004). Transcriptome analysis of the ArgR regulon in Pseudomonas aeruginosa. J Bacteriol 186, 3855-3861.

Ma, L., Jackson, K. D., Landry, R. M., Parsek, M. R. \& Wozniak, D. J. (2006). Analysis of Pseudomonas aeruginosa conditional psl variants reveals roles for the psl polysaccharide in adhesion and maintaining biofilm structure postattachment. J Bacteriol 188, 8213-8221.
Matsukawa, M. \& Greenberg, E. P. (2004). Putative exopolysaccharide synthesis genes influence Pseudomonas aeruginosa biofilm development. J Bacteriol 186, 4449-4456.

Merod, R. T., Warren, J. E., McCaslin, H. \& Wuertz, S. (2007). Toward automated analysis of biofilm architecture: bias caused by extraneous confocal laser scanning microscopy images. Appl Environ Microbiol 73, 4922-4930.

Mueller, L. N., de Brouwer, J. F., Almeida, J. S., Stal, L. J. \& Xavier, J. B. (2006). Analysis of a marine phototrophic biofilm by confocal laser scanning microscopy using the new image quantification software PHLIP. BMC Ecol 6, 1.

Nishijyo, T., Haas, D. \& Itoh, Y. (2001). The CbrA-CbrB twocomponent regulatory system controls the utilization of multiple carbon and nitrogen sources in Pseudomonas aeruginosa. Mol Microbiol 40, 917-931.

O'Toole, G. A. \& Kolter, R. (1998a). Flagellar and twitching motility are necessary for Pseudomonas aeruginosa biofilm development. Mol Microbiol 30, 295-304.

O'Toole, G. A. \& Kolter, R. (1998b). Initiation of biofilm formation in Pseudomonas fluorescens WCS365 proceeds via multiple, convergent signalling pathways: a genetic analysis. Mol Microbiol 28, 449-461.

O'Toole, G. A., Gibbs, K. A., Hager, P. W., Phibbs, P. V., Jr \& Kolter, R. (2000). The global carbon metabolism regulator $\mathrm{Crc}$ is a component of a signal transduction pathway required for biofilm development by Pseudomonas aeruginosa. J Bacteriol 182, 425-431.

Parkins, M. D., Ceri, H. \& Storey, D. G. (2001). Pseudomonas aeruginosa GacA, a factor in multihost virulence, is also essential for biofilm formation. Mol Microbiol 40, 1215-1226.

Patriquin, G. M., Banin, E., Gilmour, C., Tuchman, R., Greenberg, E. P. \& Poole, K. (2008). Influence of quorum sensing and iron on twitching motility and biofilm formation in Pseudomonas aeruginosa. J Bacteriol 190, 662-671.

Platt, M. D., Schurr, M. J., Sauer, K., Vazquez, G., Kukavica-Ibrulj, I., Potvin, E., Levesque, R. C., Fedynak, A., Brinkmann, F. S. \& other authors (2008). Proteomic, microarray, and signature-tagged mutagenesis analyses of anaerobic Pseudomonas aeruginosa at pH 6.5, likely representing chronic, late-stage cystic fibrosis airway conditions. J Bacteriol 190, 2739-2758.

Pratt, L. A. \& Kolter, R. (1998). Genetic analysis of Escherichia coli biofilm formation: roles of flagella, motility, chemotaxis and type I pili. Mol Microbiol 30, 285-293.

Recht, J. \& Kolter, R. (2001). Glycopeptidolipid acetylation affects sliding motility and biofilm formation in Mycobacterium smegmatis. J Bacteriol 183, 5718-5724.

Rice, S. A., Tan, C. H., Mikkelsen, P. J., Kung, V., Woo, J., Tay, M., Hauser, A., McDougald, D., Webb, J. S. \& Kjelleberg, S. (2009). The biofilm life cycle and virulence of Pseudomonas aeruginosa are dependent on a filamentous prophage. ISME J 3, 271-282.

Römling, U. \& Amikam, D. (2006). Cyclic di-GMP as a second messenger. Curr Opin Microbiol 9, 218-228.

Schembri, M. A., Kjaergaard, K. \& Klemm, P. (2003). Global gene expression in Escherichia coli biofilms. Mol Microbiol 48, 253-267.

Schuster, M., Hawkins, A. C., Harwood, C. S. \& Greenberg, E. P. (2004). The Pseudomonas aeruginosa RpoS regulon and its relationship to quorum sensing. Mol Microbiol 51, 973-985.

Shrout, J. D., Chopp, D. L., Just, C. L., Hentzer, M., Givskov, M. \& Parsek, M. R. (2006). The impact of quorum sensing and swarming motility on Pseudomonas aeruginosa biofilm formation is nutritionally conditional. Mol Microbiol 62, 1264-1277.

Singh, P. K., Schaefer, A. L., Parsek, M. R., Moninger, T. O., Welsh, M. J. \& Greenberg, E. P. (2000). Quorum-sensing signals indicate that 
cystic fibrosis lungs are infected with bacterial biofilms. Nature 407, 762-764.

Southey-Pillig, C. J., Davies, D. G. \& Sauer, K. (2005). Characterization of temporal protein production in Pseudomonas aeruginosa biofilms. J Bacteriol 187, 8114-8126.

Sriramulu, D. D., Nimtz, M. \& Romling, U. (2005). Proteome analysis reveals adaptation of Pseudomonas aeruginosa to the cystic fibrosis lung environment. Proteomics 5, 3712-3721.

Tümmler, B., Bosshammer, J., Breitenstein, S., Brockhausen, I., Gudowius, P., Herrmann, C., Hermann, S., Heuer, T., Kubesch, P. \& other authors (1997). Infections with Pseudomonas aeruginosa in patients with cystic fibrosis. Behring Inst Mitt (98), 249-255.

Tu Quoc, P. H., Genevaux, P., Pajunen, M., Savilahti, H., Georgopoulos, C., Schrenzel, J. \& Kelley, W. L. (2007). Isolation and characterization of biofilm formation-defective mutants of Staphylococcus aureus. Infect Immun 75, 1079-1088.

Vallet, I., Olson, J. W., Lory, S., Lazdunski, A. \& Filloux, A. (2001). The chaperone/usher pathways of Pseudomonas aeruginosa: identification of fimbrial gene clusters (cup) and their involvement in biofilm formation. Proc Natl Acad Sci U S A 98, 6911-6916.

Vijgenboom, E., Busch, J. E. \& Canters, G. W. (1997). In vivo studies disprove an obligatory role of azurin in denitrification in Pseudomonas aeruginosa and show that azu expression is under control of rpoS and ANR. Microbiology 143, 2853-2863.

Voelkner, P., Puppe, W. \& Altendorf, K. (1993). Characterization of the $\mathrm{KdpD}$ protein, the sensor kinase of the $\mathrm{K}^{+}$-translocating $\mathrm{Kdp}$ system of Escherichia coli. Eur J Biochem 217, 1019-1026.
Waite, R. D., Papakonstantinopoulou, A., Littler, E. \& Curtis, M. A. (2005). Transcriptome analysis of Pseudomonas aeruginosa growth: comparison of gene expression in planktonic cultures and developing and mature biofilms. J Bacteriol 187, 6571-6576.

Watnick, P. I. \& Kolter, R. (1999). Steps in the development of a Vibrio cholerae El Tor biofilm. Mol Microbiol 34, 586-595.

Whitchurch, C. B., Erova, T. E., Emery, J. A., Sargent, J. L., Harris, J. M., Semmler, A. B., Young, M. D., Mattick, J. S. \& Wozniak, D. J. (2002a). Phosphorylation of the Pseudomonas aeruginosa response regulator AlgR is essential for type IV fimbria-mediated twitching motility. J Bacteriol 184, 4544-4554.

Whitchurch, C. B., Tolker-Nielsen, T., Ragas, P. C. \& Mattick, J. S. (2002b). Extracellular DNA required for bacterial biofilm formation. Science 295, 1487.

Whiteley, M., Bangera, M. G., Bumgarner, R. E., Parsek, M. R., Teitzel, G. M., Lory, S. \& Greenberg, E. P. (2001). Gene expression in Pseudomonas aeruginosa biofilms. Nature 413, 860-864.

Yang, L., Barken, K. B., Skindersoe, M. E., Christensen, A. B., Givskov, M. \& Tolker-Nielsen, T. (2007). Effects of iron on DNA release and biofilm development by Pseudomonas aeruginosa. Microbiology 153, 1318-1328.

Yoon, S. S., Hennigan, R. F., Hilliard, G. M., Ochsner, U. A., Parvatiyar, K., Kamani, M. C., Allen, H. L., DeKievit, T. R., Gardner, P. R. \& other authors (2002). Pseudomonas aeruginosa anaerobic respiration in biofilms: relationships to cystic fibrosis pathogenesis. Dev Cell 3, 593-603.

Edited by: W. J Quax 